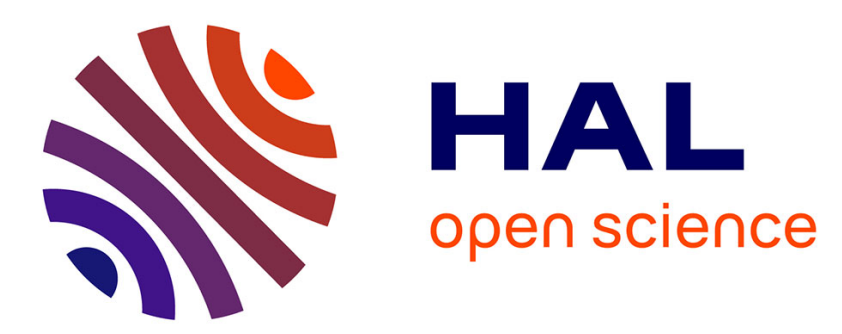

\title{
La place de l'intérêt de l'enfant dans le recours au diagnostic prénatal et au diagnostic préimplantatoire
}

Catherine Dekeuwer

\section{To cite this version:}

Catherine Dekeuwer. La place de l'intérêt de l'enfant dans le recours au diagnostic prénatal et au diagnostic préimplantatoire. Spirale, 2017, L'assistance médicale à la procréation : de l'extraordinaire aux risques du banal, 84, pp.101-112. 10.3917/spi.084.0101 . hal-01791654

\section{HAL Id: hal-01791654 \\ https://univ-lyon3.hal.science/hal-01791654}

Submitted on 27 Sep 2020

HAL is a multi-disciplinary open access archive for the deposit and dissemination of scientific research documents, whether they are published or not. The documents may come from teaching and research institutions in France or abroad, or from public or private research centers.
L'archive ouverte pluridisciplinaire HAL, est destinée au dépôt et à la diffusion de documents scientifiques de niveau recherche, publiés ou non, émanant des établissements d'enseignement et de recherche français ou étrangers, des laboratoires publics ou privés. 
Auteur : Catherine Dekeuwer

Maître de conférences en éthique contemporaine à la Faculté de philosophie, Université Jean Moulin Lyon 3. Agrégée et docteur en philosophie, licenciée de biologie moléculaire et physiologie, elle développe depuis son doctorat des méthodes de recherche en éthique appliquée, en étroite collaboration avec les professionnels de la santé. Elle travaille principalement sur les implications éthiques des recherches et des pratiques médicales qui s'appuient sur la génétique.

Catherine.dekeuwer@univ-lyon3.fr

Titre : La place de l'intérêt de l'enfant dans les décisions de recourir au diagnostic prénatal et au diagnostic préimplantatoire, Spirale, L'assistance médicale à la procréation: de l'extraordinaire aux risques du banal 2017, 84 : 101-112

Mots-clés : diagnostic prénatal, diagnostic préimplantatoire, cancers héréditaires, intérêt de l'enfant, éthique appliquée.

Les phrases clés sont soulignées dans le texte.

\section{Résumé :}

Dans les situations souvent difficiles de diagnostic anténatal, se référer à l'intérêt de l'enfant à naître peut paraître un point de repère solide. Les résultats d'une enquête menée auprès de personnes qui se savaient à risque de transmettre une anomalie génétique associée à un risque accru de cancer permet de préciser le sens et les usages possibles de cette notion.

Key words: prenatal diagnosis, preimplantation genetic diagnosis, hereditary cancers, child's interests, applied ethics.

\footnotetext{
Abstract:

The notion of child's interests seems to be very useful when the options of prenatal diagnosis and preimplantation genetic diagnosis are considered by doctors and prospective parents. This contribution presents the results of a qualitative study that investigates the way in which
} 
carriers of a genetic mutation associated with a high risk of make their reproductive decisions. It contributes to clarify, specify and refine the significations of the notion of child's interests.

\section{[Texte intégral]}

\section{Introduction}

Pour les couples qui risquent de transmettre à leurs enfants une anomalie génétique corrélée à un risque accru de maladie, recourir au diagnostic prénatal (DPN) ou au diagnostic préimplantatoire (DPI) peut sembler une solution. Ils évitent ainsi de transmettre, en même temps que l'anomalie génétique, un risque de maladie grave et toute une série de difficultés personnelles et familiales. En France, les couples qui souhaiteraient recourir au diagnostic anténatal doivent obtenir auprès d'un Centre Pluridisciplinaire de Diagnostic Prénatal (CPDPN) une attestation de gravité et d'incurabilité. L'évaluation de la sévérité de la maladie est centrale dans l'acceptabilité du recours à ces pratiques ${ }^{1}$. Cependant, elle ne fait pas toujours consensus : tous les couples n'ont pas le même point de vue sur la sévérité de la maladie qu'ils risquent de transmettre, et dans un couple, chacun des futurs parents peut avoir une évaluation différente. Il arrive aussi que les médecins qui participent aux CPDPN ne soient pas d'accord entre eux.

Dans des situations souvent compliquées, émotionnellement fortes, où les décisions sont difficiles à prendre, se référer à l'intérêt de l'enfant à naître pourrait paraître un point de repère solide. La notion d'intérêt de l'enfant a été principalement définie dans le contexte du droit de l'enfant international où elle fonctionne à la fois comme un moyen d'arbitrer entre des revendications opposées et comme une motivation pour restreindre l'exercice d'un droit (Dumortier, 2013). Dans le contexte de l'Assistance Médicale à la Procréation (AMP), l'article L. 2141-10 du Code de la santé publique dispose notamment que l'AMP peut être suspendue si l'équipe soignante estime qu'un délai de réflexion supplémentaire est nécessaire aux demandeurs «dans l'intérêt de l'enfant à naître ». D'un point de vue éthique, il paraît assez évident que les divers intérêts portés par les futurs parents et les professionnels de santé ne peuvent occuper tout l'espace de discussion, puisque le futur enfant est concerné, au premier chef, par les décisions qui sont prises. La particulière vulnérabilité de «celui qui ne

\footnotetext{
${ }^{1}$ Voir le Code de la santé publique. Articles L.2213-1 et L.2131-4, disponibles sur http://www.legifrance.fr. Le dernier rapport publié par l'Agence de la Biomédecine sur les pratiques de 2014 fait état des indications de DPN et de DPI : https://www.agence-biomedecine.fr/annexes/bilan2015/donnees/diag-prenat/03-preimpl/synthese.htm et https://www.agence-biomedecine.fr/annexes/bilan2015/donnees/diag-prenat/01-diag_prenat/synthese.htm. Pages consultées le 16 juin 2017.
} 
parle pas » (infans) induit la nécessité d'une protection lorsqu'un parent a une conception de l'intérêt de son enfant qui le met en danger ou qu'il a un intérêt propre en concurrence avec l'intérêt de l'enfant. Pourtant, dès 1989, Geneviève Delaisi de Parseval soulignait la paradoxale difficulté de prendre en compte la voix de l'enfant dans les situations d'AMP. Si la notion d'intérêt de l'enfant a « une magie propre aux notions qui recueillent un assentiment inconditionnel de la part de la société tout entière » (Dumortier, 2013, p.14), il reste que les intérêts concrets des enfants nés ou à naître restent difficile à faire valoir et aussi, tout bonnement, à définir. Des entretiens menés auprès de personnes en situation de transmettre une anomalie génétique associée à un risque accru de maladie à leurs enfants permettent d'explorer la manière dont cet intérêt est exprimé et de saisir comment il s'incarne dans ce que l'ethnographe Arthur Kleinman (1999 et 2007) appelle un «monde local d'expérience » : des controverses et compromis observables sur le terrain. Ce monde n'est pas constitué d'incantations magiques ou de concepts moraux abstraits mais de «ce qui compte vraiment» pour les acteurs.

\section{1) Description de l'enquête}

La réflexion présentée ici s'appuie sur l'analyse d'entretiens exploratoires semi-directifs menés auprès de personnes qui se savaient à risque de transmettre une anomalie génétique corrélée à un risque accru de cancer (tableau 1). Ces personnes avaient été amenées à considérer le recours au DPN ou au DPI ou étaient en cours de réflexion pour un projet d'enfant à plus ou moins long terme.

Nous avons rencontré dix personnes porteuses de mutations du gène RB1 associées au développement de tumeurs dans la rétine dans l'enfance. Quatorze entretiens concernent le cancer du sein et des ovaires dit héréditaire. Pour les porteurs d'anomalies des gènes BRCA1 ou BRCA2, le risque de développer des tumeurs est très variable, et dans certaines familles, les femmes peuvent être atteintes avant leur trentième anniversaire. Un homme nous a expliqué comment il a choisi d'avoir recours au DPI en Belgique et depuis cet entretien, au moins une attestation de gravité a été délivrée en France par un CPDPN. Les données issues de deux entretiens concernant la polypose adénomateuse familiale sont également utilisées. En l'absence de chirurgie prophylactique, le risque de cancer du côlon est estimé à $100 \%$ à 40 ans. Une surveillance est donc proposée aux enfants parfois dès l'âge de dix ans, suivie d'une colectomie quelques années plus tard. 
Ces personnes, qui connaissaient leur statut de porteur d'une anomalie génétique, ont été recrutées dans deux centres français spécialisés en génétique. S'ils le souhaitaient, leurs conjoints pouvaient participer à une partie de l'entretien. Les personnes étaient invitées notamment à nous raconter leur histoire personnelle et familiale de la maladie et à évoquer les décisions procréatives qu'elles avaient eu à prendre ou qu'elles étaient en train de prendre.

Ces entretiens suggèrent que les couples sont amenés, pour chaque projet d'enfant, à négocier une solution en fonction de leurs représentations de la sévérité de la maladie, de leurs opinions sur ce que devrait être une bonne vie de famille, des devoirs moraux qu'ils estiment avoir entre conjoints et envers leurs apparentés, de la prise en considération de ce que chacun peut supporter, et même d'un positionnement métaphysique sur le droit à intervenir dans le cours naturel des choses (Dekeuwer et Bateman, 2013). Il est tout à fait frappant de constater la diversité des positions relativement à l'acceptabilité du recours au DPN ou au DPI dans des situations comparables. Pour une même pathologie et dans des conditions comparables en termes de lieu de prise en charge, de risque de transmission, de risque de développer la maladie, du nombre de personnes malades et de décès dans la famille, de l'expression d'un désir d'enfant ou du nombre d'enfants déjà nés, les personnes peuvent considérer le recours au diagnostic anténatal comme une solution acceptable moralement ou non. S'il est difficile d'isoler un élément plus important que les autres, une préoccupation constante commande la solution qui est trouvée : comment conserver les conditions d'une vie en commun possible ? Nous analyserons ici la manière dont l'intérêt de l'enfant à naître est évoqué dans ce contexte par ceux qui aimeraient être (à nouveau) parents.

\section{2) «Il y a quelque chose de très égoïste malgré tout dans la création »}

La considération de l'intérêt de l'enfant est rarement celle qui vient en premier, et parfois elle n'est pas évoquée du tout pendant dans les entretiens. Dans ce dernier cas, le désir d'avoir un enfant ou le projet d'avoir une famille occupe tellement de place que la personne n'évoque pas le point de vue de l'enfant. Tout se passe comme si ce désir ou ce projet ne pouvait pas être remis en question. Par exemple, pour Madame A, qui a déjà un enfant atteint de phénylcétonurie ${ }^{2}$ et sait qu'elle risque de transmettre aussi une mutation du gène BRCA1,

\footnotetext{
${ }^{2}$ Un régime alimentaire très strict permet aux enfants atteints de cette maladie génétique grave de ne pas développer de retard intellectuel.
} 
c'est le projet d'avoir une famille avec plusieurs enfants qui, de toutes les façons, est le point de référence. Pendant l'entretien, elle ne se place pas du point de vue de son enfant déjà né ni du point de vue des autres enfants qu'elle souhaiterait avoir. Toutes ses décisions, qu'elles portent sur la prise en charge de sa santé, le risque de transmettre des anomalies génétiques, ou les choix professionnels, sont prises en fonction du projet - « ancré » - d'avoir une famille. Certains des couples que nous avons rencontrés n'hésitent pas à évoquer une certaine forme d' " égoïsme » de ceux qui aimeraient être parents. Par exemple, à un moment de l'entretien où Monsieur B évoque, en se plaçant au-delà de sa propre situation, l'acceptabilité morale du DPN et du DPI, il commence par constater : « il y a quelque chose de très égoïste malgré tout dans la création ». Il développe ensuite : «les mères ne veulent pas voir le risque pour leurs enfants, c'est 'Moi j'veux être mère', et elles ne voient pas les risques pour l'enfant, elles ne veulent pas les voir ». Cette critique fait écho, en philosophie, aux discussions concernant la moralité de décisions fondées sur l'égoïsme, par distinction d'avec les intérêts des autres personnes (Larrère, 2001). Pour certains soignants, le fait que les décisions procréatives se centrent sur les intérêts propres du couple ou sur l'intérêt de l'enfant revêt aussi une importance éthique, au risque même de fonder un jugement de valeur sur des couples « égoïstes » qui ne prendraient pas (suffisamment) en compte l'intérêt de l'enfant.

Nos entretiens suggèrent cependant que le caractère fondamental du projet d'enfant ou la force du désir d'être mère ne suffisent pas à expliquer le fait que certaines personnes ne se placeraient pas du point de vue de l'enfant pour considérer leurs choix procréatifs. Chez beaucoup de couples en effet, l'intérêt de l'enfant est confronté à ce qu'ils appellent euxmêmes une forme d' "égoïsme» personnel. Madame C explique qu'avant de rencontrer son mari, elle était «vraiment obtuse, à [se] dire 'je n'aurai pas d'enfant' ». Elle explique : «je ne voulais pas qu'il ait des problèmes, je ne voulais pas qu'il soit énucléé ». Elle-même, ayant développé des tumeurs dans l'enfance, a été énucléée et porte une prothèse. Le couple décide finalement de fonder une famille, mais rencontre un problème de fertilité qui le conduit à recourir à une fécondation in vitro (FIV), qui débouche, au bout de cinq années de démarches, à une grossesse. Cette femme explique qu'ils avaient prévu de recourir à une interruption médicale de grossesse (IMG) si l'enfant s'avérait porteur de l'anomalie génétique. Pourtant, à la suite d'un DPN qui indique que l'enfant est porteur, ils décident de poursuivre la grossesse : «on voulait vraiment un enfant». Leur petit garçon est malade, mais pour le moment les hospitalisations «se passent bien ». Pour un second enfant, Madame C met en balance son désir d'être à nouveau maman, les difficultés à être enceinte, et le risque que l'enfant soit malade. Elle explique: «parfois je me dis 'ah bah si, je veux un deuxième 
enfant', mais parce que je ne prends pas en compte la maladie, quoi, je prends que le désir, mon désir d'avoir à nouveau à cajoler un bébé ». Elle se sent également coupable d'avoir un enfant atteint et insiste sur le fait qu'elle pourrait se justifier auprès de son premier enfant, en lui expliquant qu'avec son mari ils souhaitaient vraiment avoir une famille. Pour un second enfant par contre, « ce serait de l'égoïsme de prendre ce risque ». Au moment de l'entretien, la décision n'était pas arrêtée.

Cet exemple, comme beaucoup d'autres, invite d'abord à penser que lorsqu'il y a dilemme entre les projets et les désirs des couples d'un côté et les intérêts d'un futur enfant de l'autre, ce dilemme ne peut pas être compris ou évalué en dehors de la temporalité propre à chaque histoire. Le jugement rétrospectif d'un déséquilibre en faveur de l'égoösme d'un parent ou du couple contre l'intérêt de l'enfant (ou inversement) peut méconnaitre ce qui lui donne sens : la temporalité d'une histoire (une rencontre, la longue attente d'une grossesse, l'expérience d'un premier enfant malade et la découverte du plaisir à être maman par exemple). S'il est possible de raconter ces histoires à partir des décisions qui sont finalement prises, les couples avancent en réalité « un pas après l'autre ». Pour Monsieur B, «avant de dire 'on pense à l'enfant', c'est déjà 'nous', parce qu'on est bien dans la personne qui va apprendre à marcher, c'est un pas après l'autre. Donc c'est d'abord nous. L'enfant ? [Il rit]... il attend, lui ! Il est rangé pour l'instant. Il attend. ». A chaque étape de cette histoire, des équilibres provisoires sont trouvés, qui permettent d'avancer. A certains moments, les intérêts des uns et des autres peuvent difficilement être séparés; ils émergent à d'autres moments lorsque certaines étapes sont franchies. Par exemple, avant même d'exister biologiquement, l'enfant est porté, constitué par le désir de ceux qui aimeraient être parents. A ce moment-là du cheminement, distinguer ce qui relève de l'intérêt de l'enfant et des intérêts du couple a-t-il un sens ? De plus, dans ce cheminement, tous les couples n'ont pas le même rapport au présent et au futur. Madame A explique qu'elle règle les problèmes les uns après les autres : "pour les maladies qui nous concernent, on essaie de ne pas se prendre la tête et on vit au jour le jour ». D'autres couples, nous y reviendrons, imaginent la vie que pourrait avoir leur enfant sur le long terme.

Le récit de Madame $\mathrm{C}$ montre aussi à quel point l'advenue d'une grossesse peut renverser une décision. Faut-il dès lors considérer que selon que l'enfant est en projet sans exister biologiquement ou que l'enfant commence à exister lors d'une grossesse, son intérêt est évalué différemment? Un embryon, un fotus et un enfant déjà né n'ont déjà pas le même statut ontologique, juridique et moral (Fagot-Largeault, 1987). En quel sens peut-on défendre les intérêts de personnes qui ne sont pas mêmes conçues? 


\section{3) Les intérêts d'une personne à venir}

La notion d'intérêt de l'enfant est souvent approchée par le biais de la considération de ses besoins fondamentaux. Ces besoins sont eux-mêmes définis à partir de ce qui semble nécessaire au développement d'un enfant : besoin d'être alimenté, de sécurité, d'abri, de soins médicaux, de stabilité, d'échanges physiques, de socialisation, d'amour et d'appartenance, de reconnaissance, etc. (Groupe d'appui à la protection de l'enfance, 2011). Dans les entretiens que nous avons menés, les couples ne se référent pas directement aux besoins des enfants à qui ils pourraient donner naissance mais plutôt à l'ensemble des problèmes qu'ils pourraient rencontrer dans leur vie du fait de leur statut de porteur. Ils envisagent les problèmes associés à la maladie, à sa prévention et à ses traitements, et aussi toute une série de difficultés personnelles auxquelles pourraient être confrontés leurs enfants. Il peut s'agir de difficultés professionnelles, liées par exemple au fait d'être aveugle. Des difficultés affectives sont aussi évoquées. Une mère d'une petite fille qui envisage une deuxième grossesse s'inquiète : non seulement ses filles risquent, comme elle, de développer des cancers du sein, mais elle pense aussi que ses filles risquent d'être rejetées et d'avoir du mal à trouver un conjoint et à fonder une famille. Certains parents aimeraient beaucoup éviter que leurs enfants soient confrontés aux choix difficiles qu'eux-mêmes ont eu à prendre : faire le test génétique, recourir à une mastectomie prophylactique, ou envisager de réaliser un diagnostic anténatal par exemple. Même dans le cas du rétinoblastome, qui touche presque exclusivement les enfants avant l'âge de cinq ans, les préoccupations de ceux qui souhaiteraient avoir un enfant concernent son devenir d'adulte. Par exemple, pour ce père qui a reçu une autorisation de recourir au DPI, il s'agit d'éviter à son enfant d'être dans la situation qu'il vit actuellement «en tant que parent » et non « en tant que porteur » puisqu'il explique que pour lui, il n'y a plus de risque de développer des tumeurs.

En dehors du contexte propre au cancer, une étude de Marie Gaille (2016) auprès de vingthuit personnes et couples rencontrés dans les jours qui ont suivi les résultats d'un DPN suggère également qu'envisager l'intérêt de l'enfant se fait dans un temps long. Les couples « s'interrogent sur ce que deviendra l'enfant, si la grossesse est menée à son terme, une fois devenu adulte. Pourra-t-il travailler? Pourra-t-il procréer? Sera-t-il autonome ? Sera-t-il heureux dans une société perçue comme intolérante au handicap et à la différence ? ».

Les préoccupations de ces couples peuvent donc bien être référées à plusieurs catégories de besoins fondamentaux : besoin d'être en bonne santé, besoins affectifs, de reconnaissance, de 
responsabilités. En ce sens, il n'est pas absurde l'utiliser la notion d'intérêt de l'enfant. Mais ceux qui aimeraient devenir parents ne considèrent pas seulement le développement de l'enfant: ils considèrent aussi l'ensemble de la vie d'adulte de leur enfant. Leurs préoccupations s'étendent parfois même aux générations futures : quelques couples souhaitent « gommer une boulette de la nature » et «briser la chaîne » qui unit les membres de leur famille au risque de développer un cancer. Dans ce contexte, le concept d'intérêt de l'enfant $\underline{\text { renvoie surtout au fait d'être porté à l'existence par des parents et non à un âge de la vie }}$ déterminé par la dépendance ou l'incapacité à choisir par soi-même. Ceci permet de résoudre la difficulté soulevée plus haut: les différents statuts ontologiques, moraux et juridiques de l'enfant en projet, de l'embryon, du fœtus, ou du bébé n'empêchent pas que ceux qui souhaiteraient être parents considèrent les intérêts d'un futur adulte, d'une personne à venir.

\section{4) « Tout faire au mieux pour tous »}

L'évaluation de l'intérêt de «la personne à venir »s'effectue principalement à l'aune de l'expérience, personnelle ou familiale, de la maladie. Il s'agit essentiellement de ne pas lui transmettre ce que l'on a soi-même subi. Un couple, qui envisageait une grossesse naturelle, raconte que la maladie du père est devenue de plus en plus sévère, et ce bien au-delà de ce qui avait été prédit par les médecins. Ces complications l'ont conduit à considérer que le DPI était non seulement préférable, mais indispensable. Dans le cas du rétinoblastome, plusieurs parents qui avaient pris le risque d'avoir un enfant porteur pour une première grossesse expliquent qu'ils n'avaient pas imaginé à quel point la maladie pouvait être grave pour leur enfant et la prise en charge très lourde. Ils ne pensaient pas que leur bébé pourrait naître avec des tumeurs dans les yeux et commencer une chimiothérapie à sept jours. Ils n'avaient pas anticipé que l'atteinte pouvait être bilatérale ni que l'enfant, selon le positionnement de la tumeur, pouvait perdre la vision centrale. Dans d'autres cas, ce sont des situations évaluées comme insupportables que les couples souhaitent éviter à leurs enfants, comme la perte d'apparentés très proches.

Préoccupés de savoir comment conserver ou rendre une vie commune possible, certaines personnes utilisent une logique d'optimisation, comme Madame F, qui risque de transmettre une anomalie du gène BRCA1 et qui voudrait tout faire pour qu'il y ait le moins de problème possible «pour tout le monde ». Monsieur D explique qu'il souhaite «simplifier la vie » de 
« tout le monde ». Il s'agit pour lui de ne pas revivre tout ce qu'ont vécu ses propres parents lorsqu'il a été malade étant enfant (les déplacements, l'inquiétude, la surveillance, l'attente). Il s'agit aussi de simplifier la vie de famille, sa compagne ayant déjà deux enfants dont ils doivent s'occuper. Cherchant aussi à simplifier « la vie qui allait venir », le recours au DPN lui parait finalement être la seule solution.

Certains choix sont donc décrits comme une sorte de calcul d'un intérêt général optimum pour toute la famille. Or, maximiser les intérêts du maximum d'individu est le mot d'ordre de la philosophie morale utilitariste, pour laquelle la seule action véritablement morale est précisément celle qui maximise les intérêts du maximum d'individu. Jusqu'où cette perspective philosophique est-elle éclairante?

\section{5) Décider « par amour»}

Le raisonnement utilitariste suppose de pouvoir isoler les intérêts de différents partis, intérêts souvent identifiés à des préférences rationnelles. Les communautés morales sont alors pensées comme des associations dans lesquelles s'opère une égale prise en compte des intérêts individuels. Cette perspective a l'avantage d'aider à résoudre des conflits d'intérêts, en considérant la nature des intérêts en jeu, en distinguant des intérêts primaires et d'autres plus secondaires et en établissant un ordre de priorité entre eux (Singer, 1993). Cependant, elle laisse de côté certains aspects importants de la délibération des couples.

Monsieur $\mathrm{F}$ explique que dans sa famille, les trois personnes qui portent une anomalie du gène RB1 ont une vie tout à fait «normale», ce qui signifie pour lui qu'elles ont un emploi et un ou plusieurs enfants. Il s'agit de sa mère, de sa sœur et de lui-même. La naissance de son premier enfant, sans recourir au diagnostic anténatal, «a été un choc » : l'enfant naît avec des tumeurs. Pour un second enfant, il explique que lui et sa compagne sont divisés. Il a une extrême réticence pour le DPI, précisément parce que sa mère, sa sœur et lui vivent tout à fait normalement. De plus, expliquer à son premier enfant, qui a développé des tumeurs, pourquoi dans son cas ils ont pris le risque de transmettre l'anomalie génétique et pas pour son frère ou sa sœur lui paraît vraiment très difficile. Sa compagne, elle, ne supporte pas de prendre le risque d'avoir un second enfant porteur, avec toute l'incertitude sur le devenir de cet enfant que cela comporte. Monsieur F explique qu'il a tranché « par amour pour V. [sa femme] » et a accepté de recourir au DPI.

Dans une perspective utilitariste, les intérêts sont définis et pondérés rationnellement, ce qui laisse peu de place aux sentiments. Or, comme Monsieur F, les couples évoquent l'importance 
des sentiments dans leurs délibérations: la culpabilité d'avoir transmis une anomalie génétique, la peur de la mort et de la maladie, celle de l'abandon aussi. La seconde limite d'une perspective utilitariste vient de ce que certaines personnes se réfèrent à un principe moral, qui s'impose quels que soient les intérêts des uns et des autres. Le contenu de ce principe diffère : « il faut éviter la maladie aux enfants » ou « il faut accepter la vie avec ses imperfections » par exemple. L'adoption de tels principes place souvent les couples dans des situations difficiles où la maximisation des intérêts est impuissante. Enfin, comparer les intérêts entre eux pour les maximiser peut conduire à réduire la complexité des situations et comporte un risque de passer à côté de la compréhension des dilemmes vécus par les couples. Considérer qu'un choix est un dilemme, c'est en effet reconnaître qu'il impose de trancher, ce qui est différent d'optimiser un ensemble d'intérêts. Le contexte décisionnel des couples conduit souvent à la reconnaissance du caractère irrémédiablement tragique de certains choix, aucune solution n'étant véritablement satisfaisante.

\section{Conclusion}

Dans les délibérations des couples que nous avons rencontrés et qui risquent de transmettre une anomalie génétique associée à un risque accru de cancer, la notion d' «intérêt de l'enfant » renvoie à l'ensemble des difficultés qu'il risque de rencontrer, y compris et surtout dans sa vie d'adulte, du fait de son statut de porteur d'une anomalie génétique. Se placer du point de vue de la personne à venir paraît bien être une dimension importante du choix des parents prospectifs.

La notion d'intérêt de la personne à venir pourrait donc bien constituer un point de repère lorsque des décisions difficiles sont à prendre, y compris pour les professionnels de santé. Amener les couples à adopter ce point de vue lorsque cela n'est pas spontané ou aider à pondérer les différents intérêts constitue souvent, pour les équipes médicales que nous avons rencontrées dans le cadre de cette recherche, un aspect de la pratique de soin. Les résultats présentés ici permettent d'abord de souligner que porter plus attention au cheminement des couples permettrait d'éviter une opposition trop simple, et parfois moralisatrice, entre les intérêts d'un couple et ceux de la personne à venir. Ils permettent également de mieux comprendre ce qui relève de l'intérêt de la personne à venir du point de vue des couples, qui 
n'est réductible ni à l'ensemble des critères médicaux qui permettent d'évaluer le risque de maladie et la qualité de vie ni aux normes sociales qui définissent l'intérêt de l'enfant ${ }^{3}$.

Les résultats présentés invitent aussi à poser une question. L'expérience vécue de la maladie et de ses conséquences influence considérablement l'évaluation par les couples de l'intérêt de la personne à venir. Mais certains couples regrettent une forme de naïveté dans leur anticipation de la maladie. Dans certains parcours de soin, les professionnels de santé essaient de fournir le plus d'information possible aux parents. Faudrait-il s'attacher à mieux faire se rencontrer l'expérience et la connaissance des professionnels de santé et cette expérience vécue?

Enfin, nos entretiens témoignent du fait que très souvent les intérêts de chaque membre de la famille (qui dépassent largement les considération médicales) sont pondérés dans une délibération qui vise ce qu'il y a de mieux, ou de plus simple, ou de plus acceptable «pour tout le monde ». Cependant, cette pondération ne suffit pas à décrire ni à comprendre les délibérations des couples, qui confrontent parfois ces intérêts avec des sentiments et des principes moraux. Elle ne rend pas compte non plus de la dimension proprement tragique de certains choix, qui ne peut être résolue dans la détermination d'un intérêt général. Finalement, même si la notion d'intérêt a une certaine efficacité pour évaluer des conflits d'intérêt, elle ne suffit pas, d'un point de vue éthique, à comprendre les décisions que doivent prendre les couples.

\section{Remerciements}

Cette recherche a été financée par la Fondation de France et l'Agence de la biomédecine. Je remercie chaleureusement les personnes qui ont accepté de nous rencontrer, les équipes médicales qui nous ont accueillies, et ma collègue Simone Bateman, qui a participé à l'étude.

\section{Bibliographie}

DEKEUWER, C. BATEMAN, S. 2013. «Much more than a gene : hereditary breast and ovarian cancer, reproductive choices and family life », Medicine, Health Care and Philosophy, $16(2)$ p. $231-244$

\footnotetext{
${ }^{3}$ Dumortier (2013) en cite une liste à partir de la jurisprudence, qui montre bien que ces normes sociales relèvent du sens commun, peuvent rentrer en conflit, et contiennent souvent des présupposés normatifs implicites.
} 
DELAISI DE PARSEVAL, G. 1989. «Procréations artificielles et intérêt de l'enfant », Etudes, 370/2, p.173-185

DUMORTIER, T. 2013. "L'intérêt de l'enfant : les ambivalences d'une notion protectrice », Journal du droit des jeunes, $\mathrm{n}^{\circ} 329, \mathrm{p} .13-20$

FAGOT-LARGEAULT, A. 1987. «Les droits de l'embryon humain et la notion de personne humaine potentielle », Revue de Métaphysique et de Morale, n³, p.361-385

GAILLE, M. 2016. «Des terres morales inconnues. Du diagnostic prénatal à la décision de poursuivre ou d'interrompre une grossesse », Anthropologie médicale, 12 | 2016, mis en ligne le 30 mai 2016. https://anthropologiesante.revues.org/1991, page consultée le 12 juin 2017 GROUPE D'APPUI A LA PROTECTION DE L'ENFANCE. 2011. La notion d'intérêt de l'enfant dans la loi réformant la protection de l'enfance. Texte disponible sur www.reformeenfance.fr, page consultée le 16 juin 2017.

KLEINMAN, A.1999. "Moral Experience and Ethical Reflection: Can Ethnography Reconcile Them? A Quandary for «The New Bioethics » », Daedalus, Vol.128, n4, p.69-97 KLEINMAN A. 2007. What Really Matters. Living a moral life admist uncertainty and danger, Oxford University Press Marcus GE

LARRERE C. 2001. " Intérêt. Intérêt et égoïsme », in CANTO-SPERBER M. (dir) Dictionnaire d'éthique et de philosophie morale, Paris, PUF

SINGER, P. 1993. Practical Ethics, Cambridge, Cambridge University Press 
Tableau 1 : Données concernant les personnes rencontrées

\begin{tabular}{|c|c|c|c|}
\hline & Rétinoblastome & $\begin{array}{l}\text { Cancer du sein et des } \\
\text { ovaires }\end{array}$ & $\begin{array}{l}\text { Polypose } \\
\text { adénomateuse } \\
\text { familiale }\end{array}$ \\
\hline \multirow[t]{2}{*}{ Sexe } & 7 hommes & 2 hommes & 2 hommes \\
\hline & 3 femmes & 12 femmes & \\
\hline \multirow[t]{2}{*}{ Etat civil } & 10 mariés ou en couple & 13 mariés ou en couple & $\begin{array}{l}2 \text { mariés ou en } \\
\text { couple }\end{array}$ \\
\hline & & 1 célibataire & \\
\hline $\begin{array}{l}\text { Aucun } \\
\text { enfant }\end{array}$ & 2 & 6 & 1 \\
\hline 1 enfant & 5 & $\begin{array}{l}6 \text { (dont deux femmes } \\
\text { enceintes du second enfant) }\end{array}$ & 1 \\
\hline 2 enfants & 3 & 2 & \\
\hline $\begin{array}{l}\text { Recours } \\
\text { au DPN }\end{array}$ & $\begin{array}{l}4 \text { DPN (une IMG, une } \\
\text { naissance d'un enfant } \\
\text { porteur, naissance de } \\
\text { deux enfants non } \\
\text { porteurs). }\end{array}$ & & \\
\hline $\begin{array}{l}\text { Recours } \\
\text { au DPI }\end{array}$ & $\begin{array}{l}1 \quad(5 \text { essais, pas de } \\
\text { naissance }) \text { et deux } \\
\text { dossiers en cours } \\
\text { d'examen. }\end{array}$ & $\begin{array}{l}2 \text { (naissance de deux } \\
\text { enfants) }\end{array}$ & 1 (une naissance) \\
\hline
\end{tabular}

